\title{
Aleatory Epitope Recognition Prevails in Human T Cell Responses?
}

\author{
Paul V. Lehmann* \& Alexander A. Lehmann \\ Research and Development Department, Cellular Technology Limited (CTL), Shaker Heights, OH, 44122 \\ *Address all correspondence to: Paul V. Lehmann, Research and Development Department, Cellular Technology Limited (CTL), Shaker Heights, \\ OH, 44122; Tel.: +1 216-791-5084; Fax: +1 216-751-1373, E-mail: paul.lehmann@immunospot.com
}

\begin{abstract}
Eli Sercarz pioneered epitope recognition by T cells. Studying mice, he made the seminal observation decades ago that epitope dominance is so unpredictable with mixed MHC haplotypes that he coined it aleatory, for dice-like. Accordingly, for every individual there is a unique potential epitope space that is defined by the polymorphic and polygenic MHC molecules (restriction elements) expressed. Of this potential epitope space, some peptides will elicit stronger $\mathrm{T}$ cell responses than others, bringing about the actually realized epitope space. The selection of the actually recognized peptides from the potential epitope space is random, however, resulting in unique epitope dominance and hierarchy patterns in individuals. Engaging in brute-force epitope scans, which permit the assessment of the entire potential epitope space at the highest possible resolution, we observe aleatory epitope recognition in human CD8 cell responses to viruses. Because the selection of peptide has fundamental implications for successful $\mathrm{T}$ cell immune monitoring, we dedicate this article to Eli Sercarz in a special issue of Critical Reviews ${ }^{\text {TM }}$ in Immunology in his honor.
\end{abstract}

KEY WORDS: ELISPOT, brute-force epitope mapping, immune dominance, epitope prediction, $\mathrm{T}$ cell repertoire, ImmunoSpot

ABBREVIATIONS: CEF, peptide pool consisting of CMV, EBV, and FLU epitopes; EBV, Epstein-Barr virus; ELISPOT, enzyme-linked ImmunoSpot assay; H-2, histocompatibility system 2 gene complex, murine MHC; HCMV, human cytomegalovirus; HEL, hen egg white lysozyme; HLA, human leukocyte antigen gene complex, human MHC; MHC, major histocompatibility gene complex; PBMC, peripheral blood mononuclear cell; PVDF, polyvinylidene fluoride

\section{INTRODUCTION}

All scientists stand on the shoulders of the giants who taught them. For me (PVL), Eli Sercarz was one of these giants, (Zoltan A. Nagy being the other), to whom I owe major credit. I am one of those blessed immunologists whose child has not been repelled by experiencing the craze of two scientist parents' daily life (or perhaps the lack of a life beyond science) and who also chose to follow the same path. By standing on my shoulders, my son, Alex (AL), therefore also stands on Eli's. Alex's PhD thesis is in a field that Eli pioneered, immune monitoring in general and $\mathrm{T}$ cell epitope/determinant recognition in particular. In this article, which has been written in honor of Eli Sercarz, father and son summarize Eli's legacy as it surfaces almost daily in our discussions of Alex's brute force determinant/epitope mapping data.

\section{THE LEGACY OF ELI SERCARZ}

\section{A. In Vivo Veritas}

I selected Eli's laboratory at UCLA for my second postdoctoral training because at that time it was leading the attempt to understand $\mathrm{T}$ cell-mediated immunity in vivo. I should add that I had already committed to this field of research and had become intrigued by Eli's dreamy and warmhearted personality, having met him for the first time during his visit to Zoltan's lab, where I did my first postdoctoral training. I joined Eli in 1989 and stayed with him until 1993. That was at a time when mainstream immunological research had already (prematurely) switched from in vivo studies of immunity to in vitro investigations at the cellular and molecular levels, including the initial attempts to define the rules of peptide binding to MHC alleles. Although we also 
participated in pioneering such studies, Eli constantly remined us that "in vivo veritas" (playfully referring to the old Roman saying "in vino veritas" for "in wine lies the truth"), that any effort to increase understanding of molecular details needs to be coupled with verifying whether and how these contribute to immune function as a whole.

In the context of $\mathrm{T}$ cell-mediated immunity, we of course needed to learn what the rules are for MHC-peptide binding, but that knowledge, as Eli kept on emphasizing, does not permit predicting whether a peptide capable of MHC binding in the test tube will actually be generated through natural antigen processing in vivo and, if it does whether it will be able to outcompete other peptides in the antigen-presenting cell (APC) for binding to MHC molecules, and if so which of the several available MHC molecules will capture a peptide. To complicate matters further, even if a peptide is presented, its presence or abundance on the surface of the APC will not permit conclusions about the characteristics of the peptide-specific pre-immune $\mathrm{T}$ cell repertoire capable of responding to this peptide - that is, the breadth or diversity of this peptide-specific naïve $\mathrm{T}$ cell repertoire, the affinity distributions of the individual $\mathrm{T}$ cell receptors (TCRs) within this repertoire, and the precursor frequency of each of these specificities that in itself is regulated by positive and negative selection processes that are unique for individuals in an outbred population.

Eli kept reminding us that, even if we knew about a peptide being presented and about the availability of a peptide-specific naïve $\mathrm{T}$ cell repertoire, we would not be able to predict the magnitude or the quality of the ensuing $\mathrm{T}$ cell response, not even to that single peptide determinant, not to mention the entire antigen or microorganism. These ideas have been captured in hundreds of Eli's original contributions to this topic and are reviewed in Sercarz et al. ${ }^{1}$ I fondly remember pondering these ideas with him at length on the veranda of his lovely home in Topanga Canyon. A bottle of his favorite Chianti frequently accompanied discussions that lasted into the late hours of the mild California nights to remind us of the wisdom of "in vivo and in vino veritas."

\section{B. Aleatory Determinant Recognition}

As captured in his review article, ${ }^{1}$ back then Eli had already shown that $\mathrm{T}$ cells are prone to respond to several peptides on even small model antigens, and we considered this to be the rule for $\mathrm{T}$ cell recognition of complex microbial or self-antigenic systems. He established that only in inbred parental mouse strains (that show limited $\mathrm{MHC} / \mathrm{H}-2$ restriction element diversity, being monozygotic for $\mathrm{H}-2$ haplotypes) is the $\mathrm{T}$ cell response hierarchy to several epitopes of an antigen predictable and consistent. In F1 mice, in which restriction element diversity increases because of heterozygosity (still far from, but closer resembling that of humans) not only does the diversity of epitopes to which $\mathrm{T}$ cell responses are generated increase, as one might expect, but the actual $\mathrm{T}$ cell recognition of these epitopes becomes highly variable and unpredictable in individuals. ${ }^{2}$ The likely interpretation for this finding is that, when several potential H-2 restriction elements are present, including new hybrid combinations not present in the parents, these compete for peptide binding.

Variations in the proteolytic enzyme levels in APCs and the $T$ cell repertoire are an additional factor that can add to the heterogeneity of response. Accordingly, one needs to distinguish between the potential epitope space, representing all peptides that possibly could be presented in an individual based on the MHC alleles present in that individual, and the expressed epitope space, representing those peptides against which that individual actually mounts a $\mathrm{T}$ cell response. Each individual therefore responds to only a fraction of the potential epitope space, and the selection of peptides for his/her expressed epitope space is apparently random. In these murine models, the selection of actually recognized determinants was observed to be so erratic that Eli coined the term "aleatory determinant recognition" (alea means dice in Latin). ${ }^{3}$

Aware of all these complexities, Eli was calling into question the wisdom of reducing immune monitoring in humans to a single (or a few) peptide determinants, because one can assess the magnitude and quality of antigen-specific $\mathrm{T}$ cell immunity only by measuring the $\mathrm{T}$ cell response to all peptide determinants/epitopes that $\mathrm{T}$ cells target on an antigen. 
To reliably detect the antigen-specific $\mathrm{T}$ cells in any given individual, either one needs to know the expressed epitope space and use the corresponding peptide(s) for the detection of the $\mathrm{T}$ cells, or one needs to test "agnostically" by providing any possible peptide for T cell recall and just "see" instead of assuming. Because, according to Eli, the expressed epitope space cannot be predicted for humans, we chose for Alex's project the agnostic path. Doing so permits us to unambiguously establish whether or not it is valid to base immune monitoring efforts in humans on assessing a single (or a few) epitopes/ restriction elements - for example, by using multimers such as tetramers, pentamers, and dextramers.

\section{Dynamic Determinant Recognition}

At the time, had already introduced the notion that $\mathrm{T}$ cell determinant hierarchy in any given individual is not necessarily a fixed feature of immunity; rather, it can be dynamic. ${ }^{4}$ For example, if peptide A on a "foreign" antigen is dominant in triggering a $\mathrm{T}$ cell response, it will also excel in inducing self-tolerance if that protein is a "self" antigen, sparing only the low-affinity end of the A-reactive T cell repertoire. ${ }^{5}$ Similarly, if peptide A on a foreign antigen (or a neoepitope of a tumor) is more immunogenic than peptide $B$, that will lead early on in the immune response to the dominance of high-affinity A-reactive $\mathrm{T}$ cells. However, should the antigen persist, it will also likely lead to the preferential exhaustion of the high-affinity A-reactive T cell repertoire. In that case, $\mathrm{T}$ cells recognizing determinant $\mathrm{A}$ with low affinity or determinant $\mathrm{B}$ will dominate the $\mathrm{T}$ cell response at later time points. ${ }^{6}$ Early on, Eli was emphasizing to us how important it is to consider such basic concepts while selecting peptides for studying $\mathrm{T}$ cell immunity in chronic infections, autoimmunity, and antitumor immunity. Only recently, with frustration growing about the inaccuracy of in silico predictions of the expressed epitope space (not the potential epitope space, however), have we seen how accurate Eli's predictions were. ${ }^{7}$

On that veranda in Topanga Canyon, we were pondering such concepts with Eli and brainstorming how to test them in mice. And then, dreaming of one day being able to address the same questions directly for humans, we were back to studying mice in the lab.

\section{Eli's Spots}

We clearly understood that progress in understanding $\mathrm{T}$ cell immunity in humans would depend on developing new technologies for reliably detecting and studying the rare peptide-specific $\mathrm{T}$ cells among the overwhelming majority of $\mathrm{T}$ cells that are specific for other antigens. Much of my initial effort in Eli's lab focused on developing and comparing such techniques, eventually leading to my life-long love affair with ELISPOT.

It was Alex Miller, Eli's long-time assistant lab director, who brought the potential of the ELISPOT technique to my attention: no matter how rare an antigen-specific $\mathrm{T}$ cell is, its antigen-triggered secretory cytokine signature should be detectable when the cells are seeded on a surface that is coated with an anticytokine capture antibody. Moreover, it should be possible to count the number of antigen-triggered cytokine-producing $\mathrm{T}$ cells, thus establishing their frequency in a primary test sample (e.g., freshly isolated lymph node or spleen cells, or peripheral blood mononuclear cells, PBMCs), thereby measuring the magnitude of the expressed $\mathrm{T}$ cell repertoire that is specific to the antigen/peptide. Moreover, by detecting the different cytokines that these T cells secrete, one can learn about their effector lineage (i.e., Th1, Th2, Th17, Th21) and thus the quality of the antigen-specific $\mathrm{T}$ cell response.

This all was theory in those early days. The problem we faced was that the original ELISPOT protocols were developed for the detection of antibody-secreting B cells (ASCs) and relied on polystyrene for analyte capture. ${ }^{8,9}$ However, ASCs secrete about 100 times more antibody molecules per minute than do $\mathrm{T}$ cells secreting cytokine molecules. The surfaces used, polystyrene first and then nitrocellulose, do not have sufficient capture antibody-binding capacity for reliable $\mathrm{T}$ cell detection. Here and there were reports of successful $\mathrm{T}$ cell ELISPOT implementations, but the assay was considered so unreliable back then that its reputation was like that of "cold fusion." Nevertheless, believing in the potential of $\mathrm{T}$ cell ELISPOT, for years we 
in Eli's lab tried to get this assay reliably working for the detection of antigen-specific T cells ex vivo, but without much success.

Only years later, after having tested virtually every variable, did we find the solution in the polyvinylidene fluoride (PVDF) membrane. The PVDF binds capture antibodies (Abs) via hydrophobic interactions resulting in their stronger adhesion than by the electrostatic forces that underlie capture $\mathrm{Ab}$ binding to polystyrene or nitrocellulose. ${ }^{10}$ Moreover, PVDF has a fractal surface that results in much denser membrane coating and thus better-defined spots. ${ }^{11}$ The difference we observed was as "day and night" in the ability to detect individual interferon gamma (IFN- $\gamma$-) - secreting T cells (Fig. 1) and also in the reproducibility of test results. ${ }^{12}$ Of note, in the first report of ELISPOT for T cell detection, which relied on the nitrocellulose membrane, the same type of poorly defined spots were published (see Fig. 1 in Czerkinsky et al. ${ }^{13}$ ), resulting in barely interpretable data.

Our introduction of the PVDF membrane was the birth hour of modern $T$ cell ELISPOT analysis, and the resolution gained immediately enabled

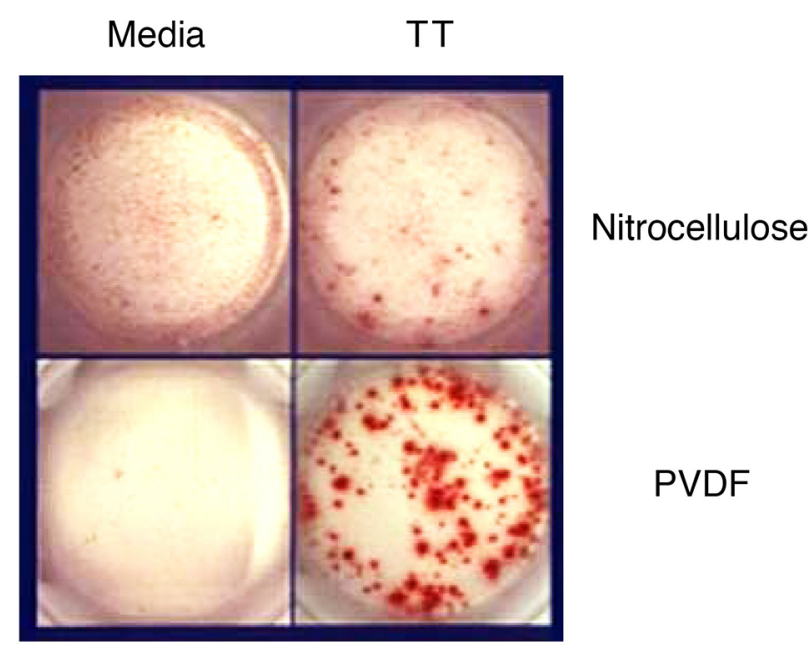

FIG. 1: Interferon gamma-detecting ELISPOT assay on nitrocellulose versus PVDF membrane. Tetanus toxoid (TT-)-induced production of the cytokine versus its spontaneous release (media) was tested on human PBMCs. Except for the membrane, all other assay conditions were identical. These are the images of our first membrane comparison, done in 1995 , on which the patent was based. major discoveries. ${ }^{14,15}$ I was already at Case Western Reserve University by then, but having started the effort in Eli's lab, we frequently called the assay "Eli's spots" in Eli's honor.

ELISPOT has evolved into a mature tool for $e x$ vivo $\mathrm{T}$ cell analysis, and I have contributed some 200 publications to this field. The detection limit of flow cytometry is reached at $0.01 \% .{ }^{16}$ Peptide-specific $\mathrm{T}$ cells in PBMC, however, typically occur in frequencies lower-frequently much lower - than that. In contrast, ELISPOT's detection limit is 1 in 1 million, ${ }^{17}$ which extends the window of observation 100 times toward the detection of antigen-specific $\mathrm{T}$ cells. In addition, ELISPOT requires many fewer PBMCs than flow cytometry, ${ }^{18}$ and the data analysis can be automated. ${ }^{19}$ All these features make ELISPOT ideal for high-resolution, high-throughput $\mathrm{T}$ cell testing. ${ }^{20}$ My son Alex has been spearheading the effort to establish the logistics for testing hundreds and even thousands of peptides on a single human donor. ${ }^{21}$

Enabled by technological progress, we can now systematically address the type of questions that we started to investigate in Eli's day but now in humans (accounting for the extensive MHC polymorphisms and polygenism in the outbred population versus the few MHC alleles expressed by inbred mice). We are studying real antigens (e.g., entire viral proteins rather than small model antigens) that trigger $\mathrm{T}$ cell responses via naturally occurring infections (as opposed to their subcutaneous injections with complete Freund's adjuvant, CFA).

Following "Elian" questioning, we can now unambiguously determine (1) how many peptides on complex viral antigens have induced CD8 T cell responses in different human subjects; (2) whether these peptides can be predicted based on the HLA Class I alleles expressed by the study subject; (3) how many of the predicted peptides are actually recognized; (4) whether, if donors share a Class I allele (e.g., HLA A-2), they will predictably develop CD8 cell responses to shared peptides; and if so (5) whether there are shared dominant, subdominant, and cryptic determinants; in other words, is there a conserved determinant hierarchy? Knowing the answers to these questions is essential for T cell immune monitoring. When immune monitoring is 
conducted with, for example, multimers (tetramers, pentamers, and dextramers), individual peptides presented by one of the Class I alleles in a test subject are assumed to be representative for the CD8 T cell response in that subject. Is such an approach to immune monitoring warranted at all?

\section{E. Complete Brute-Force Determinant Mapping}

To be able to unambiguously answer the questions just listed, it is important to test every possible peptide of the antigen that could be recognized by the test subject's CD8 T cell system. As the peptide-binding groove on Class I molecules is closed on both ends and accommodates only peptides of 8-10 amino acids in length, it does not tolerate frame shifts. ${ }^{22}$ Only a peptide library that walks the sequence of a protein in steps of single amino acids (Fig. 2) will systematically provide all peptides that might be recognized by CD8 $\mathrm{T}$ cells in any given individual, presented by the different Class I molecules expressed by that individual. Such peptide libraries therefore permit querying the entire potential epitope space. Only a few of these peptides will recall CD8 T cells ex vivo - those that elicited a $\mathrm{T}$ cell response, including clonal expansion in vivo. Nine-mer peptide libraries that progress in steps of two amino acids would already miss half of the epitopes recalled by single peptides, and detect reliably only the determinants revealed by adjacent peptides. ${ }^{21}$ Systematic bruteforce CD8 T cell-determinant mapping with 9-mer peptides and single amino acid overlays, however, involves testing hundreds of individual peptides for every test subject. Until recently, such an undertaking seemed illusory given (1) the number and cost of the peptides involved, (2) the number of PBMCs needed per test subject, and (3) the investigator time needed to execute the experiment and analyze the data. No longer, as we will see.

With his pioneering spirit, Eli was the first to implement systematic determinant mapping. ${ }^{23}$ Back then, we studied the CD4 T cell response to hen egg white lysozyme (HEL). HEL is a relatively small enzyme consisting of a single polypeptide of 129 amino acids in length. Because the peptide-binding groves of Class II molecules are open on both ends, they are tolerant to frame shifts, which permitted the use of longer peptides and progression of the sequence in larger steps. ${ }^{24}$ The scale of CD4 T cell epitope mapping for HEL was therefore a fraction of what we needed to do for systematic CD8 T cell mapping of viral antigens in humans. Still, the principle was the same and was introduced by Eli.

\section{F. Aleatory Viral Antigen Recognition in Humans}

Using the brute-force approach, we studied the fine specificity of the $\mathrm{T}$ cell repertoire specific for human

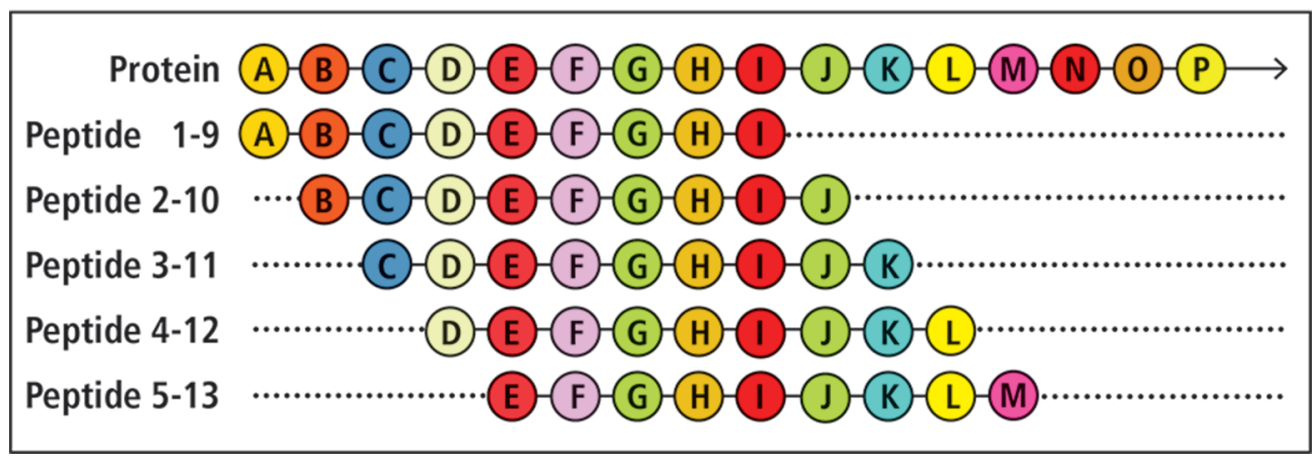

FIG. 2: Principle of brute-force CD8 cell epitope mapping. A library of 9-aa-long peptides is created that walks the antigen's sequence in steps of single amino acids. By systematically testing all of these peptides one by one, the entire potential epitope space is covered. Peptides that have induced a CD8 cell response in vivo resulting in clonal expansions can be detected in PBMCs ex vivo via the increased frequency of peptide-reactive cytokine-producing CD8 cells. 
cytomegalovirus (HCMV) in humans who had or had not been infected with this virus. ${ }^{21}$ The published data were confined to HLA-A*02:01-positive subjects (who differed in all other Class I alleles). In HCMV-seronegative subjects, none of the HCMV peptides triggered a recall response, suggesting that cross-reactive/promiscuous peptide recognition by $\mathrm{T}$ cells is not a confounding factor in monitoring $\mathrm{T}$ cell immunity to HCMV (see Table 1 in P. Lehmann et al. $\left.{ }^{21}\right)$. In contrast, in all HCMV-seropositive individuals' PBMCs, HCMV peptide-reactive T cells were detected in high frequency (see Table 1 in P. Lehmann et al. ${ }^{21}$ ). Therefore, the specificity of the ELISPOT test system used was exquisite.

The fine specificity of HCMV recognition by $\mathrm{T}$ cells was surprising, however. For each donor, we tested peptide pools that systematically covered 20 open reading frames of HCMV (15-mer peptides progressing the respective protein antigen's amino acid sequence in steps of 11 amino acids). There was no immunodominant antigen in the cohort (see Table 1 in P. Lehmann et al. ${ }^{21}$ ). Antigen HCMV-X, which was immunodominant in subject $\mathrm{A}$, would be barely, if at all, targeted in subject B, who would in turn respond vigorously to antigen HCMV-Y (and mostly to several other HCMV antigens as well). In individual $\mathrm{C}, \mathrm{HCMV}-\mathrm{X}$ was subdominant with yet other HCMV antigens dominating. The antigen recognition pattern within the cohort seemed to be aleatory. We made similar observations when studying recognition of the individual protein antigens that constitute EBV (see Supplementary Table 2 in P. Lehmann et al. ${ }^{21}$ ) and influenza virus (A. Lehmann, unpublished). These data reveal that $\mathrm{T}$ cell immune monitoring that relies on a single viral antigen is likely to largely underestimate the magnitude of the expressed virus-specific $\mathrm{T}$ cell repertoire, even missing it altogether in some individuals. Seeing these data, Eli's wisdom resounds: "Sometimes you need to step back so you can see better."

\section{G. Aleatory Viral Epitope Recognition in Humans}

The previously mentioned HCMV proteome-wide studies, carried out with pools of 15-mer peptides, detected CD4 T cells and, to some extent, CD8 T cells. Using such longer peptide pools made the broad scope of the interrogation feasible but its precision less than ideal. While 15 mers are well suited to binding with HLA Class II molecules and thus to $\mathrm{CD} 4 \mathrm{~T}$ cell recall, they cannot bind directly to HLA Class I molecules for CD8 T cell recall. Such longer peptides can activate CD8 T cells only after cross-presentation, or proteolytic cleavage, or when shorter peptide fragments arise as byproducts of peptide synthesis. Large gaps in detection of the CD8 $T$ cell repertoire can also be expected when using overlapping 15-mer peptides with 11-aa-long gaps covering the protein sequence because of the HLA Class I molecules' intolerance of frame shifts. We therefore created a 9-mer peptide library that covered the HCMV pp65 sequence in steps of single amino acids, providing every possible determinant for CD8 T cell recognition (Fig. 2). Each of these peptides, (553 in total) was tested individually on the PBMCs of five test subjects, all of whom were seropositive for HCMV and expressed the HLA-A02:01 allele.

Among the peptides tested was one, pp65(495503), that is considered immunodominant in HLA-A02:01-positive subjects. ${ }^{25}$ Because of its alleged immune dominance, this peptide, also called CEF-7, has been included in the CEF peptide pool to serve as a positive control for the functional detection of human CD8 T cells. ${ }^{26}$ Raw data of such an ELISPOT assay performed by AL are shown in Fig. 3. This donor shows a marginal response-in Eli's terminology, a cryptic response - to pp65(495503)/CEF-7 (present in well A3 as a control on this plate). Of note, pp65(495-503)/CEF-7 was highly active, as it induced vigorous recall responses in other test subjects in the same experiment (see Table 2 in P. Lehmann et al. ${ }^{21}$ ). While it did not respond to pp65(495-503)/CEF-7, the HLA-A02:01 allele-positive subject shown in Fig. 3 mounted a vigorous recall response to two adjacent peptides, pp65(417-425) and pp65(418-426), revealing an epitope with the 418-426 core. Please note the exquisite signal-to-noise resolution of the assay, which is also illustrated in Supplementary Fig. 5 in P. Lehmann et al. ${ }^{21}$

The pp65(495-503)/CEF-7 peptide was indeed found to be immunodominant in two of the 


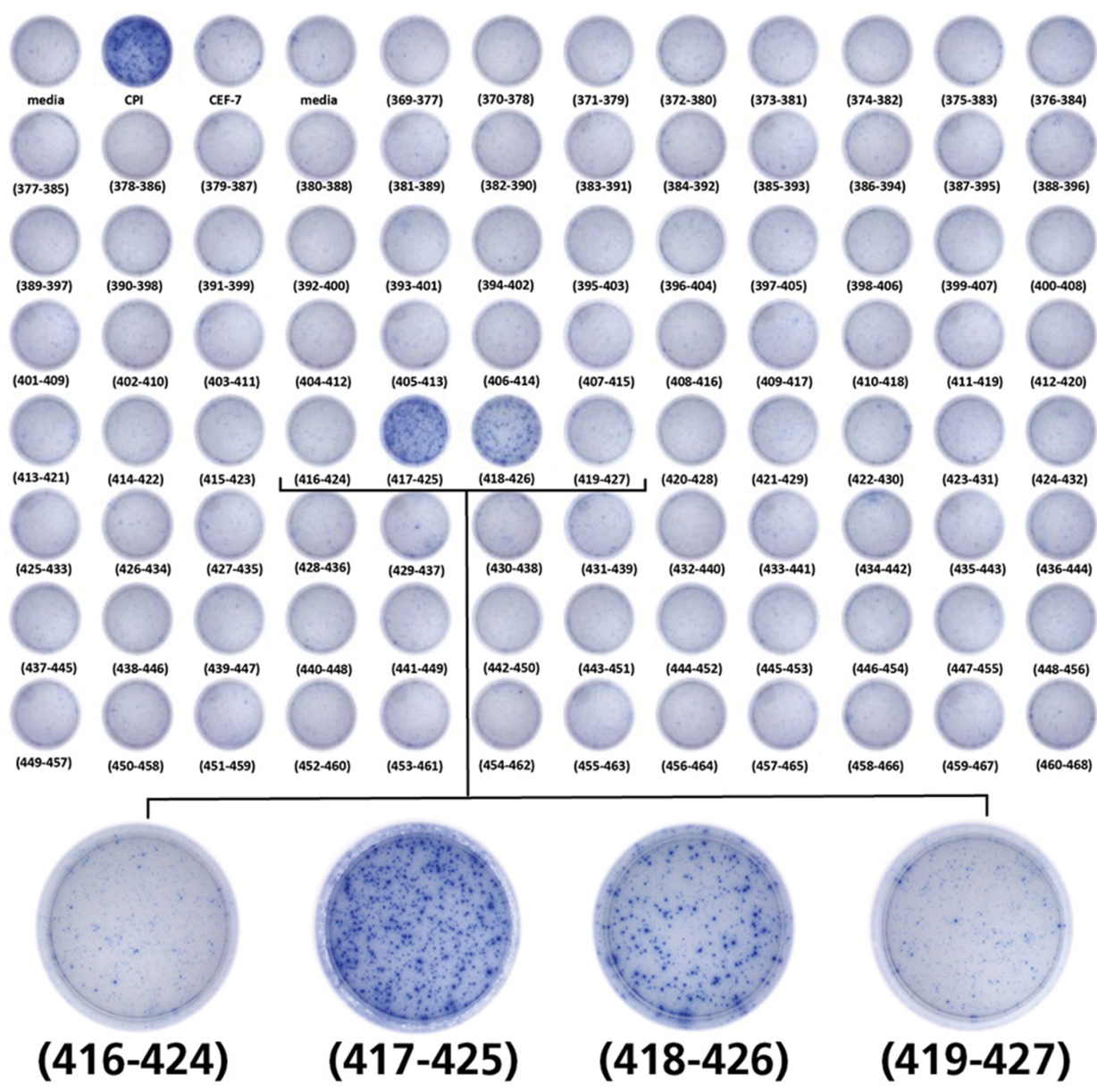

FIG. 3: Brute-force epitope mapping test: images of a 96-well ImmunoSpot plate. All wells of the plate contain PBMCs from the same HLA-A02:01-positive HCMV-seropositive donor with 300,000 cells plated per well (6 plates actually tested per donor to accommodate the $553 \mathrm{HCMV} 9$-mer peptides of which one plate is shown). Controls include media-only wells (A1 and A4), CPI as positive control (A2), and the pp65(495-503)/CEF7 peptide considered immune dominant in such subjects. ${ }^{33}$ Wells A5-H12 contain the specified HCMV nonamer peptides. The peptide-triggered IFN- $\gamma$ production is detected at single-cell resolution in an ImmunoSpot assay. Magnified images for the two positive and adjoining negative wells are shown at the bottom.

five test subjects (Table 2 in P. Lehmann et al. ${ }^{21}$ ). In two of the five HLA-A02:01-positive test subjects, it was co-dominant, just one among several peptides that recalled CD8 T cells in a comparable frequency range (Table 2 in P. Lehmann et al. ${ }^{21}$ ). The non-pp65(495-503) 9-mer peptides targeted by CD8 T cells were scattered in an apparently aleatory fashion over the pp65 molecule. Therefore, it was not only impossible to predict which of the many antigens of HCMV (and possibly of any other virus) would be preferentially targeted by $\mathrm{T}$ cells of any given individual (Table 1 in P. Lehmann et al. ${ }^{21}$ ); even within an HLA allele-matched cohort, a single (or even a few) "dominant" peptide(s) could not be relied on to detect and characterize the antigen-specific CD8 T cell repertoire (Table 2 in P. Lehmann et al. ${ }^{21}$ ). This phenomenon does not appear to be unique to HCMV. For EBV and influenza as well, we have shown that previously defined "immune dominant peptides" frequently fail to reveal the respective virus-specific memory $\mathrm{T}$ cell pool ${ }^{27}$; we have made similar observations for hepatitis B virus also. ${ }^{6}$ 


\section{H. Predicted vs. Realized Epitopes}

After having completed the first assessment of the pp65-specific CD8 cell repertoire in five HLA-A*02:01-positive subjects, ${ }^{21}$ and while AL is gearing up to extend such studies to more humans, we are starting to analyze whether the actually recognized peptides, and the magnitude of the CD8 cell response to each one, could have been predicted based on their MHC-binding properties. This analysis also addresses the question whether peptide binding scores tailored to the MHC alleles expressed in each individual permit narrowing in on a few peptides in the context of each restriction element-for example, when selecting multimers in silico for immune monitoring.

While we still are at the start of this journey, the answer is beginning to crystallize. Realized CD8 cell epitopes are rarely the top predicted binders for each of the up to 6 HLA Class I alleles expressed in an individual; they are mostly not even in the top 10 predictions for each allele. The potential epitope space is therefore considerably wider than anticipated. From this sizable potential epitope space, only a select few peptides elicit a CD8 cell response and do so in an apparent aleatory manner. Response hierarchies do not match peptide-binding hierarchies. As Eli predicted, peptides need to bind to MHC molecules, but the strength of this binding is only one of many factors determining the outcome of the $\mathrm{T}$ cell response. Looking at such data, I remember and share with AL the discussions on immune dominance I had with Eli, who would have loved to see such clear data on human $\mathrm{T}$ cell recognition. Eli postulated that, if aleatory determinant recognition occurs even with simple model antigens in mice with only a few MHC-restriction elements, it is even more likely to occur in humans with complex antigens and multiple restriction elements involved. If variability is the rule even with young age- and sex-matched, genetically identical rodents housed in the same controlled environment, how much more variability might one expect for human $\mathrm{T}$ cell responses involving a much higher degree of genetic and environmental disparity? Eli was right, and I wish he were still among us so I could tell him...

\section{A VIRTUAL RE-ENCOUNTER BETWEEN THE MASTER AND HIS DISCIPLE 27 YEARS AFTER LAST MEETING}

I am driving my sky-blue 1978 VW Bug Convertible, the very car I drove back when I worked in Eli's lab, a car I still own and occasionally drive on fine days like this. Alex sits next to me, and the two of us enjoy, top down, the commute that Eli took daily from UCLA to Topanga Canyon. As we arrive, Eli welcomes us and leads us to his dreamy veranda, where we sit down in the shade of tropical plants. It is peaceful, serene, like heaven on Earth, or is it Earth in heaven? Eli pours a glass of his favorite Chianti for us and addresses me:

Eli Sercarz (ES): Who is this handsome young man with you, Paul?

Paul V. Lehmann (PVL): My son, Alex. Remember, Magda was pregnant with him when I left. He has become an immunologist, too. I told him a lot about you; I wanted him to meet you.

ES: It has been a while, Paul. I remember well the long discussions we had on this very veranda about $\mathrm{T}$ cell recognition. We shared the passion back then.

PVL: You infected me, Eli. I am still suffering from it. I infected Alex.

ES: But I also taught you there is more to life than just science and success. You will need to tell me more about that later. Tell me first, what new did you learn about $\mathrm{T}$ cell immunity since we last met?

PVL: Eli, remember how we both were dissatisfied with the signal-to-noise performance of the proliferation assays we used back then? They were not well suited to a close look at antigen-specific T cells ex vivo, but that was all we had.

ES: Yes, I remember well, and also our discussions on the need to come up with a better readout. You were quite passionate about it, Paul. You nearly ruined my lab with the costs of your assay development efforts. Did you eventually succeed in coming up with something better?

PVL: Eli, remember how we were betting on ELISPOT, but just did not get it to work 
back then? All it took was a change of membrane. Look for yourself: this is the clarity of data it provides now (I show him Fig. 1). This is one of your pepscans, testing human PBMC (I show him Fig. 3). Look at the strength of signal induced by the positive peptides, and how low to nonexistent the background is with all the negative peptides. We have verified that we are detecting individual peptide-specific CD8 $\mathrm{T}$ cells here! We can do even fourcolor assays now, learning about CD8 $\mathrm{T}$ cell sublineages (I proudly show him Hanson et al. $\left.{ }^{28}\right)$ !

ES: This is amazing! 553 peptides plus controls on a single human?

PVL: That was just a warmup. Alex is gearing up to test 14,000 individual peptides for bruteforce epitope scans of coronavirus, including all of its mutations! All 14,000 peptides tested on individual donors!

ES: You are dreaming! Not even an elephant has enough PBMCs for such tests!

PVL: With ELISPOT, that just takes 1.4 billion PBMCs if we go for the regular 96-well format. By leukapheresis we can obtain ten times more cells from a single donor at each draw! Using 384 well plates, we would need one-third as many cells ${ }^{18}-470$ million PBMCs. If pressed even more for cells, we could go for the matrix approach while still identifying individual peptides. ${ }^{29}$ That could further cut down the PBMCs needed-for example, 4.8-fold to 98 million PBMCs, obtainable from $100 \mathrm{~mL}$ of blood.

ES: That means you could do genome-wide epitope scans?

PVL: That's what we are doing for coronavirus, including testing for all known mutations.

ES: But that means you now can get direct answers to all the questions we tried to address in murine models!

PVL: Yes, Eli. Now we can get crystal-clear answers for most foreign antigen-specific $\mathrm{T}$ cell responses. We are even progressing with self-antigens. (I show him Przybyla et al..$^{30}$ ).
ES: OK. So update me on foreign antigens. We always hoped there would be immune dominance. It would make life so much easier for immune monitoring if it were the rule. Take a virus, with all its proteins. Is one of the antigens preferentially recognized by $\mathrm{T}$ cells over the others? Is there a response hierarchy?

PVL: We looked at CMV (I show him Table 1 in P. Lehmann et al. ${ }^{21}$ ) and EBV (I show him Supplementary Table 2 in P. Lehmann et al. $\left.{ }^{21}\right)$. For these two viruses, the answer is crystal-clear: there is no immune dominant antigen. Alex has similar, unpublished data for influenza, and he is gearing up to ask this very question for coronavirus. Presently it looks as if antigen dominance does exist; this is the exception, not the rule.

ES: This is bad news for those who bet on individual antigens for immune monitoring. But I am not surprised. It reminds me of our aleatory $\mathrm{T}$ cell recognition data in mice. So, what about immune dominant determinants?

PVL: Same thing. (I show him Table 2 in P. Lehmann et al. ${ }^{21}$ ).

ES: I would have expected to see it...

PVL: On a mere theoretical basis perhaps, me, too. But the data are crystal-clear. Many times a determinant-say an A-2-restricted peptide that is dominant in one A-2-positive human - can even be cryptic in another A-2-positive human (I point out the pp65[495-503] peptide, well A3, in Fig. 3, and the other data in Table 2 in P. Lehmann et al. ${ }^{21}$ With a smile, I add) It is aleatory recognition, Eli.

ES: This might be unique to CMV. What about other viruses?

PVL: Same for influenza and EBV (I show him Moldovan et al. ${ }^{27}$ ). Soon Alex will tell us about coronavirus.

ES: But what about determinant predictions? We understood the rules of MHC-peptide binding quite well back then?

PVL: Those rules hold up well. All peptides that are actually targeted by $\mathrm{T}$ cells can be verified to bind to one or several HLA alleles expressed by the test subject. The problem is 
that many more peptides match predictions than are actually recognized by $\mathrm{T}$ cells, and that recognition of the actual peptides is aleatory among humans. Do I need to remind you, Eli, about your reminding us all the time how peptide display on an MHC allele is a necessary step but just one of many leading to a $\mathrm{T}$ cell response?

ES: But brute-force systematic testing of individual peptides is barely an option for general $\mathrm{T}$ cell immune monitoring...

PVL: Dozens, possibly hundreds, of peptides can be pooled, Eli, without much interference. ${ }^{31}$

ES: Propagating this notion will not make you popular among in silico and multimer folks, Paul.

PVL: Data are data, Eli, and these are crystal clear. In vivo et in vino veritas. Eventually the truth will prevail. Our determinant-spreading story did not make me a favorite either back in our days together, ${ }^{4}$ when everyone wanted to cure autoimmune diseases based on the assumption that there is immune dominance in the autoimmune $\mathrm{T}$ cell response. Now it is textbook knowledge, and cancer vaccine trials are based on determinant spreading. As to in vivo versus in silico, I like how Alex Sette puts it: "If you can get a picture of something, you can discuss whether you like it or not but if there's no picture there's nothing to discuss." ${ }^{32}$

ES: Yes, Paul, we had much fun together back then. But now let science be science, and tell me how you fared since we last met. I see you still drive the same little convertible....

Perhaps Eli and I will have such an encounter in a heavenly setting one day, and I hope there is Chianti up there, too. I wonder whether Alex's coronavirus data will modify my update to Eli...

\section{REFERENCES}

1. Sercarz EE, Lehmann PV, Ametani A, Benichou G, Miller A, Moudgil K. Dominance and crypticity of T cell antigenic determinants. Annu Rev Immunol. 1993;11:729-66.

2. Moudgil KD, Wang J, Yeung VP, Sercarz EE. Heterogeneity of the T-cell response to immunodominant determinants within hen eggwhite lysozyme of individual syngeneic hybrid $\mathrm{F}_{1}$ mice: Implications for autoimmunity and infection. J Immunol. 1998;161:6046-53.

3. Sercarz EE. The architectonics of immune dominance: The aleatory effects of molecular position on the choice of antigenic determinants. Chem Immunol. 1989;46:169-85.

4. Lehmann PV, Forsthuber T, Miller A, Sercarz EE. Spreading of T-cell autoimmunity to cryptic determinants of an autoantigen. Nature. 1992;358:155-7.

5. Targoni OS, Lehmann PV. Endogenous myelin basic protein inactivates the high avidity T-cell repertoire. J Exp Med. 1998;187:2055-63.

6. Anthony DD, Valdez H, Post AB, Carlson NL, Heeger PS, Lehmann PV. Comprehensive determinant mapping of the hepatitis C-specific CD8 cell repertoire reveals unpredicted immune hierarchy. Clin Immunol. 2002;103:264-76.

7. Brennick CA, George MM, Srivastava PK, Karandikar SH. Prediction of cancer neoepitopes needs new rules. Sem Immunol. 2020 Feb;47:101387.

8. Czerkinsky CC, Nilsson LA, Nygren H, Ouchterlony O, Tarkowski A. A solid-phase enzyme-linked Immunospot (ELISPOT) assay for enumeration of specific antibody-secreting cells. J Immunol Methods. 1983;65:109-21.

9. Sedgwick JD, Holt PG. A solid-phase immunoenzymatic technique for the enumeration of specific antibody-secreting cells. J Immunol Methods. 1983;57:301-9.

10. Wahlgren M, Arnebrant T. Protein adsorption to solid surfaces. Trends Biotechnol. 1991;9:201-8.

11. Weiss AJ. Overview of membranes and membrane plates used in research and diagnostic ELISPOT assays. Methods Mol Biol. 2012;792:243-56.

12. Zhang W, Caspell R, Karulin AY, Ahmad M, Haicheur N, Abdelsalam A, Johannesen K, Vignard V, Dudzik P, Georgakopoulou K, Mihaylova A, Silina K, Aptsiauri N, Adams V, Lehmann PV, McArdle S. ELISPOT assays provide reproducible results among different laboratories for T-cell immune monitoring-even in hands of ELISPOT-inexperienced investigators. J Immunotoxicol. 2009;6:227-34.

13. Czerkinsky C, Andersson G, Ekre H-P, Nilsson L- $\AA$, Klareskog L, Ouchterlony Ö. Reverse ELISPOT assay for clonal analysis of cytokine production I. Enumeration of gamma-interferon-secreting cells. J Immunol Methods. 1988;110:29-36.

14. Lehmann PV, inventor; Case Western Reserve University, assignee. Methods for measuring T-cell cytokines. United States patent 6410252. $1995 \mathrm{Dec} 22$

15. Forsthuber T, Yip HC, Lehmann PV. Induction of TH1 and TH2 immunity in neonatal mice. Science. 1996;271:1728-30.

16. Romero P, Cerottini JC, Speiser DE. The human T cell response to melanoma antigens. Adv Immunol. 2006;92:187-224.

17. Hesse MD, Karulin AY, Boehm BO, Lehmann PV, Tary-Lehmann M. A T-cell clone's avidity is a function of its activation state. J Immunol. 2001;167:1353-61. 
18. Hanson J, Sundararaman S, Caspell R, Karacsony E, Karulin AY, Lehmann PV. ELISPOT assays in 384-well format: Up to 30 data points with one million cells. Cells. 2015;4:71-83.

19. Zhang W, Lehmann PV. Objective, user-independent ELISPOT data analysis based on scientifically validated principles. Methods Mol Bio. 2012;792:155-71.

20. Lehmann PV, Zhang W. Unique strengths of ELISPOT for T-cell diagnostics. Methods Mol Bio. 2012;792:3-23.

21. Lehmann PV, Suwansaard M, Zhang T, Roen DR, Kirchenbaum GA, Karulin AY, Lehmann A, Reche PA. Comprehensive evaluation of the expressed CD8+ T-cell epitope space using high-throughput epitope mapping. Front Immunol. 2019;10:655-68.

22. Pamer E, Cresswell P. Mechanisms of MHC class I-restricted antigen processing. Ann Rev Immunol. 1998;16:323-58.

23. Maizels RM, Clarke JA, Harvey MA, Miller A, Sercarz EE. Epitope specificity of the T-cell proliferative response to lysozyme: Proliferative T-cells react predominantly to different determinants from those recognized by B cells. Eur J Immunol. 1980;10:509-15.

24. Cresswell P. Invariant chain structure and MHC class II function. Cell. 1996;84:505-7.

25. Wills MR, Carmichael AJ, Mynard K, Jin X, Weekes MP, Plachter B, Sissons JG. The human cytotoxic T-lymphocyte (CTL) response to cytomegalovirus is dominated by structural protein pp65: Frequency, specificity, and T-cell receptor usage of pp65-specific CTL. J Virol. 1996;70:7569-79.

26. Currier JR, Kuta EG, Turk E, Earhart LB, Loomis-Price L, Janetzki S, Ferrari G, Birx DL, Cox JH. A panel of MHC class I restricted viral peptides for use as a quality control for vaccine trial ELISPOT assays. J Immunol Methods. 2002;260:157-72.

27. Moldovan I, Targoni O, Zhang W, Sundararaman S, Lehmann PV. How frequently are predicted peptides actually recognized by CD8 cells? Cancer Immunol Immunother. 2016;65:847-55.

28. Hanson J, Roen DR, Lehmann PV. Four color ImmunoSpot assays for identification of effector T-cell lineages. Methods Mol Bio. 2018;1808:51-62.

29. Anthony DD, Lehmann PV. T-cell epitope mapping using the ELISPOT approach. Methods. 2003;29:260-9.

30. Przybyla A, Zhang T, Li R, Roen DR, Mackiewicz A, Lehmann PV. Natural T cell autoreactivity to melanoma antigens: Clonally expanded melanoma-antigen specific CD8 + memory T cells can be detected in healthy humans. Cancer Immunol Immunother. 2019;68:709-20.

31. Zhang W, Moldovan I, Targoni OS, Subbramanian RA, Lehmann PV. How much of virus-specific CD8 $T$ cell reactivity is detected with a peptide pool when compared to individual peptides? Viruses. 2012;4: 2636-49.

32. La Jolla Institute for Immunology. Detailed analysis of immune response to SARS-CoV-2 bodes well for COVID-19 vaccine. MedicalXpress [Internet]. 2020 May 15 [cited 2020 May 19]. Available from: https://medicalxpress. com/news/2020-05-analysis-immune-response-sars-covbodes.html.

33. Schiller A, Zhang T, Li R, Duechting A, Sundararaman S, Przybyla A, Kuerten S, Lehmann PV. A positive control for detection of functional CD4 T cells in PBMC: The CPI pool. Cells. 2017;6:47-58. 
